\title{
TAGUNG
}

\section{Wie viel Integration verträgt Europa?}

\author{
Michael Weiner*
}

Das „Nein“ der französischen und der niederländischen Bevölkerung zum europäischen Verfassungsvertrag war nicht rein innenpolitisch motiviert. Vielmehr bestand auch die Sorge, von einem ,EU-Superstaat " vereinnahmt zu werden. Juristisch gesehen ist die Europäische Union kein Staat und diese Sorge somit unbegründet. Politisch ist diese Befürchtung jedoch nicht völlig von der Hand zu weisen. Daher beschäftigte sich der Arbeitskreis Europäische Integration e.V. im Rahmen seines Jahreskolloquiums aus politik-, rechtsund wirtschaftswissenschaftlicher Sichtweise mit der Thematik einer vermeintlich überbordenden europäischen Dimension, die von den Bürgerinnen und Bürgern als problematisch empfunden wird und somit zum Hindernis der europäischen Integration geworden ist.

\section{Europa in der Wahrnehmung der Bürgerin-} nen und Bürger

Die deutschen Medien sind in Brüssel sehr präsent: Von circa 1200 akkreditierten Journalisten stammen 150 aus Deutschland, berichtete Norbert Robers. Darunter haben vor allem die großen deutschen Tageszeitungen eigene Brüssel-Korrespondenten, während Regionalzeitungen häufig gemeinsame ,Pools' bildeten, für die jeweils ein Korrespondent tätig sei. Die Berichterstattung über die Europäische Union gestalte sich gegenwärtig eher schwierig, da die großen Themen fehlten, die es in der Zeit der Osterweiterung und des abgelehnten Verfassungsvertrags noch gegeben habe. Auch zur Schaffung einer europäischen Öffentlichkeit trage die Presse momentan wenig bei. Die Berichterstattung über Europa erfolge

\section{Superstaat EU?}

Jahreskolloquium des Arbeitskreises

Europäische Integration e.V. mit Unterstützung der Europäischen Kommission

Berlin, 15./16. November 2007

\section{Begrüßung und Einführung}

Prof. Dr. Dr. h.c. Peter-Christian MÜLLERGRAFF, Vorstandsvorsitzender des Arbeitskreises Europäische Integration e.V.; Universität Heidelberg

Die Europäische Union im Spiegelbild der Medien

Norbert ROBERS, Redakteur, Nordwest-Zeitung, Oldenburg

Beschränkungen oder Wandel der nationalen Handlungsspielräume in der EU am Beispiel der Verkehrspolitik: Transformation Europäischer ,Governance“

Dr. Dieter PLEHWE, Wissenschaftszentrum Berlin

Europäische Rechtsetzung - überbordend oder funktionsadäquat?

Prof. Dr. Dr. h.c. Peter-Christian MÜLLERGRAFF, Universität Heidelberg

Kompetenzausweitung der EU durch externe Einbindung

Prof. Dr. Michèle KNODT, Technische Universität Darmstadt

Beschränkungen oder Wandel nationaler Handlungsspielräume in der EU? Der Wandel der parlamentarischen Funktionen

Dr. Andreas MAURER, Stiftung Wissenschaft und Politik, Berlin

* Michael Weiner, Praktikant in der Forschungsgruppe EU-Integration, Stiftung Wissenschaft und Politik, Berlin. 
noch immer aus einem stark nationalen Blickwinkel. Auch aus praktischen Beweggründen werde meist über die nationalen Abgeordneten und Funktionsträger berichtet, da es so keine Sprachbarriere gebe und ein Interview auch schneller zu führen sei. Oftmals werde auch die Tragweite der auf europäischer Ebene gefällten Entscheidungen für die eigene Leserschaft nicht erkannt. Somit fehlten auf den Regional- und Lokalseiten der Zeitungen die Nachrichten zum Thema Europa - doch gerade diese Seiten würden am meisten gelesen. Daher müsse, damit das Thema Europäische Union kein Elitethema bleibt, die Berichterstattung aus Brüssel so ,heruntergebrochen“ werden, dass die Folgen der europäischen Entscheidungsprozesse für die Lebenswelt der Leserinnen und Leser nachvollziehbar und somit interessant werden.

\section{Europäische Rechtssetzung und mitglied- staatliche Souveränität}

In seinem Vortrag berichtete Dieter Plehwe von einem Forschungsprojekt, das die Beschränkung nationaler Handlungsspielräume durch europäische Rechtssetzung im Bereich der Verkehrspolitik untersuchte. Zu einer solchen Beschränkung seien einige Behauptungen im Umlauf, zum Beispiel, dass 80 Prozent des deutschen Rechts europäischen Ursprungs sei. Ansatzpunkt der vorgestellten Studie war das Fehlen beziehungsweise die Überarbeitungsbedürftigkeit der quantitativen Erfassung und wissenschaftlichen Berechnung, um das tatsächliche Verhältnis von europäischer und nationaler Gesetzgebung zu beurteilen. Es stelle sich nämlich zum Beispiel die Frage, was überhaupt als Gesetz zähle, welche Art von Gesetzen ins Verhältnis gesetzt werden könnten und ob alle Gesetze gleich zu gewichten seien. Darüber hinaus sei zu hinterfragen, ob ein quantitativer Anstieg europäischer Gesetzgebung auch zwangsläufig einen großen supranationalen Einfluss bedeute. In der empirischen Arbeit für diese Studie zur
Steuerpolitik in der Europäischen Politik

Prof. Dr. Clemens FUEST, Universität zu Köln

Solving the Common Pool Problem in the EU Fiscal Constitution

Dr. Friedrich HEINEMANN, Zentrum für Europäische Wirtschaftsforschung, Mannheim

Europäische Wettbewerbspolitik am Scheideweg? Chancen und Risiken des more economic approach

Prof. Dr. André SCHMIDT, European Business School, Oestrich-Winkel

Verkehrspolitik sei klar geworden, dass man zunächst einmal ein tieferes Verständnis für das zu untersuchende Politikfeld entwickeln müsse, um überhaupt alle relevante Rechtssetzung zu finden. Zusammenfassend sei zu den Ergebnissen zu sagen, dass die Europäisierung zweifelsohne und in zunehmendem Maße die nationale Autonomie bei der Rechtssetzung und die der beteiligten Akteure begrenze. Zu warnen sei aber vor allgemeinen und vereinheitlichenden Äußerungen, die schnell zur Über- aber auch zur Unterschätzung der Bedeutung europäischen Rechtes führen könnten.

Anhand eines Fallbeispiels thematisierte Peter-Christian Müller-Graff die Frage der Qualität europäischer Rechtssetzung und stellte zunächst fest, dass eine neue Form des Sekundärrechts entstünde. Die ,Geschäftspraktiken Richtlinie' (2005/29/EG), ${ }^{1}$ die zum Zweck der Erhöhung des Verbraucherschutzniveaus das Verbot unerlaubter Geschäftspraktiken zum Ziel habe, bediene sich einer abstrakten und sehr umfangreichen Sprache, die rechtsaktspezifische Definitionen schaffe. Das erschwere die Übernahme in nationales Recht und die Rechtsanwendung. Die Kritik am europäischen Rechtssystem sei vielfältig. Unter anderem führe eine Zersplitterung europäischen Rechts, zum Beispiel durch mehrere parallele Richtlinien, auch zur Zersplitterung

1 Richtlinie 2005/29/EG des Europäischen Parlaments und des Rates vom 11. Mai 2005 über unlautere Geschäftspraktiken im binnenmarktinternen Geschäftsverkehr zwischen Unternehmen und Verbrauchern, in: Amtsblatt der EU, Nr. L 149 vom 11. Juni 2005, S. 22-39. 
nationalen Rechts. Außerdem bedingten viele Definitionskataloge innerhalb der Rechtsakte eine Künstlichkeit der Begriffsbildung, die allerdings bisweilen unvermeidlich sei, da es sonst zu Begriffsvermengung käme. Angesichts dieser Kritikpunkte habe die Kommission drei Aufgaben. Sie müsse zum einen supranationales Recht schaffen, das das Funktionieren des Binnenmarktes sichert. Des Weiteren müsse sie transnational verständliches und zwischenstaatlich sinnfälliges Recht schaffen. Letztendlich solle man keine zu hohen Erwartungen an eine europäische Rechtsordnung haben, denn zwangsläufig bleibe Vertrautes auf der Strecke. Allgemein präge Gemeinschaftsrecht einen neuen europäischen Normstil, der allerdings funktionsadäquat sein müsse.

Vergemeinschaftung durch Internationale Organisationen und die Rolle der Parlamente

Michèle Knodt stellte die Ergebnisse eines Forschungsprojektes vor, in dessen Rahmen sie die Auswirkungen, die der internationale Kontext (am Fallbeispiel der Welthandelsorganisation) auf das europäische Regieren hat, die Mechanismen die diese bewirken und wie sie zu bewerten sind, untersucht hatte. Zu Beginn stellte Knodt die Hypothesen auf, dass die Einbindung der Europäischen Union in das Welthandelsorganisations-System zu einem institutionellen Wandel führe, was eine Aufwertung der Gemeinschaft und die Zentralisierung des politischen Systems der Europäischen Union bedeute. Im Primärrecht sei eine Zunahme von exklusiven Kompetenzen der Union gegenüber geteilten Kompetenzen mit den Mitgliedstaaten erkennbar. Im Sekundärrecht werde durch die externe Einbindung einerseits neues Recht geschaffen, andererseits bestehendes Recht den Erfordernissen der externen Ebene angepasst. Durch Anpassungsdruck und durch Lernprozesse sei in der formalen Organisation der Union ein Wandel in Richtung Vergemeinschaftung zu erkennen. Auch in den Arbeitsroutinen und den Konzepten legitimer Ordnung der Union seien Veränderungen erkennbar. Hier gehe der Trend vor allem zu einer besseren Zusammenarbeit mit Wirtschaftsakteuren und der Einbindung von Vertretern der Zivilgesellschaft analog zur Einbindungsstrategie der Welthandelsorganisation. Letztendlich werde die Tendenz zur Gemeinschaftsförderung in der Stärkung der Rolle der Kommission deutlich.

Die schleichende und sachwidrige Zentralisierung der Regelungskompetenzen in Brüssel sei, so Andreas Maurer, ein viel bemühter Vorwurf. Wäre dies wirklich der Fall, müsse eine Bedeutungszunahme des Europäischen Parlaments erkennbar sein. Tatsächlich sei eine kontinuierliche Zunahme der Beteiligung des Europäischen Parlaments an Entscheidungen zu verzeichnen. Ohne die Gemeinsame Agrarpolitik, bei der das Europäische Parlament keine Mitsprache habe, betrage die Beteiligungsquote an Rechtsakten 90 Prozent. Die Folgen dieses Funktionswandels seien jedoch vielfältig. So sei eine Straffung und Informalisierung der Entscheidungsprozesse zu beobachten - Effizienz komme vor Transparenz. Auch sei die Auswahl der Kommissionsmitglieder und die Haushaltskompetenz ein eigenständiges Element sanktionsmächtiger Vorabkontrolle geworden. Der Funktionswandel äußere sich auch immer mehr durch die Bildung von Großkoalitionen im Parlament. Dies stünde dem traditionellen Politikverständnis der Bürgerinnen und Bürger von ,Rechts“ und ,Links“ entgegen. Die Politikgestaltungsfunktion der nationalen Parlamente hingegen werde durch zunehmende Gewaltenverschränkung und die Exekutivlastigkeit der europäischen Ebene in Frage gestellt. Die Kontrollfunktion könne häufig nur über den Umweg der europäischen Ebene wahrgenommen werden. Des Weiteren bescherten Kompetenzkonflikte, zum Beispiel zwischen AuBen- und Europaausschuss im Deutschen Bundestag, der nationalen Exekutive größeren Handlungsspielraum. Somit ergäben sich auf beiden Ebenen Lücken in der parlamentarischen Arbeit, die unter anderem zu größerer Bürgerferne führten. 


\section{Regulierung im Binnenmarkt}

Über die Reformperspektive einer gemeinsamen, konsolidierten Bemessungsgrundlage zur Unternehmensbesteuerung in der Steuerpolitik der Europäischen Union berichtete Clemens Fuest. Die Unternehmensbesteuerung sei eigentlich Sache der Mitgliedstaaten, doch dürfe sie wiederum nicht die Grundfreiheiten des Binnenmarktes gefährden. Grenzüberschreitende Vorgänge dürften daher nicht diskriminiert werden. Momentan gebe es zur Unternehmensbesteuerung 27 unterschiedliche, nationale Steuersysteme. Dies führe zu hohen administrativen Kosten für grenzüberschreitend tätige Unternehmen. Außerdem bestünde der Anreiz zur Verlagerung von Buchgewinnen in Niedrigsteuerländer. Nationale Maßnahmen zum Schutz des nationalen Steueraufkommens stünden dabei häufig in Konflikt mit den Beschränkungs- und Diskriminierungsverboten im Binnenmarkt. Momentan würden zum Zweck der Unternehmensbesteuerung die Gewinne für Mutter- und Tochterunternehmen in jedem Mitgliedstaat getrennt ermittelt. Dies führe zu großem Aufwand, da grenzüberschreitende Transaktionen im praktisch gleichen Unternehmen kompliziert abgewickelt werden müssen. Die Reformidee sehe die Ermittlung eines europaweiten, konsolidierten steuerpflichtigen Gewinns vor, der dann nach Indikatoren der realwirtschaftlichen Tätigkeit auf die Mitgliedstaaten der Europäischen Union zur Versteuerung aufzuteilen sei. Eine Angleichung der Steuersätze sei dabei nicht vorgesehen. Die ökonomischen Vorteile eines solchen Systems hielten sich jedoch in Grenzen, sodass Fuest dem Reformansatz wenig Chancen auf Verwirklichung einräumte.

\section{Das Dilemma kollektiver Entscheidungsfin- dung}

Einen Weg aus dem ,Common-Pool-Problem' versuchte Friedrich Heinemann aufzuzeigen. Das Common-Pool-Problem äußere sich durch die asymmetrische Kosten-NutzenVerteilung, die durch den Finanzrahmen der
Europäischen Union entstünde. Die Kosten würden von allen getragen, während der Nutzen über Politiken wie die Gemeinsame Agrarpolitik oder die Kohäsionspolitik lokal konzentriert werde. Dadurch entstünde für Akteure der hohe Anreiz, bei den Verhandlungen zum Finanzrahmen nicht an das, große Ganze' zu denken, sondern für die eigene Region etwas herausholen zu wollen. Dabei befänden sich die Akteure in einem Gefangenendilemma zu Lasten der Gesamtheit der Steuerzahler. Dieses Problem verschlimmere sich zudem, je höher die Anzahl der Verhandlungsparteien sei. Es sei weder durch eine EU-Steuer noch durch Mehrheitsentscheidungen im Rat oder eine Verlagerung des Verfahrens ins Europäische Parlament zu lösen. Andere Lösungen wie ein mit großen Kompetenzen ausgestatteter europäischer Finanzminister seien unrealistisch. Erfolg versprechend und gleichzeitig realistisch sei hingegen die Zweiteilung des europäischen Budgets. In einen Topf 1 kämen die Mittel für die Gemeinsame Agrarpolitik und die Kohäsionspolitik, in Topf 2 die Mittel für andere öffentliche Güter. Durch einen generellen Korrekturmechanismus stiegen die Mittel in Topf 2 relativ zu denen in Topf 1 , weshalb die Common-Pool-Problematik gleichzeitig an Gewicht verlöre.

\section{Die Rolle industrieökonomischer Modelle in der Wettbewerbspolitik}

André Schmidt legte in seinem Vortrag die Chancen und Risiken des, More Economic Approach ' dar. Diesem Ansatz zufolge sollten die Entscheidungen der Europäischen Kommission auf der Basis industrieökonomischer Modelle und daraus abgeleiteter Simulationsstudien getroffen werden. Mit dem Ziel der Konsumentenwohlfahrt solle dies vor allem im Rahmen von Einzelfallentscheidungen mit einer Ausrichtung dieser Entscheidungen an deren ökonomischen Konsequenzen geschehen. Dadurch würde eine hohe Treffsicherheit gewährleistet und Fehler minimiert werden. Der Ansatz berge allerdings auch Risiken. Da Einzelfallentscheidungen einen gewissen Er- 
messensspielraum voraussetzten, seien die Einflussmöglichkeiten der jeweiligen Lobby höher und die Rechtssicherheit geringer. AuBerdem sei eine Vielzahl von Einzelentscheidungen mit steigendem Verfahrensaufwand und höheren Kosten verbunden. Zusätzlich ergäben sich Probleme bei der Auswahl der einzusetzenden ökonomischen Modelle. Als alternatives Leitbild schlug Schmidt den ,More Order Economic Approach“ vor. Dabei solle ökonomische Theorie zu sinnvollen Regeln führen, und an den Voraussetzungen, nicht den Ergebnissen des Wettbewerbs orientiert sein. Die individuellen Handlungs- und Entschließungsfreiheiten könnten so gesichert werden.
Fazit

Die auf dem Jahreskolloquium vorgestellten Projekte und diskutierten Fragen aus verschiedenen Bereichen der Sozialwissenschaften lassen eine zweigeteilte Bilanz der Entwicklung der Europäischen Union zu. Einerseits konnte gezeigt werden, dass bestehende Behauptungen, die Europäische Union habe sich widerrechtlich zu einem Superstaat entwickelt, nicht der Realität entsprechen. Dennoch wurde auch deutlich, dass die AuBenwahrnehmung der Europäischen Union verbesserungsbedürftig ist. Zudem entfernt sich das Europäische Parlament von den europäischen Bürgerinnen und Bürgern.

\title{
Die europäische Integration aus deutsch-französischer Perspektive
}

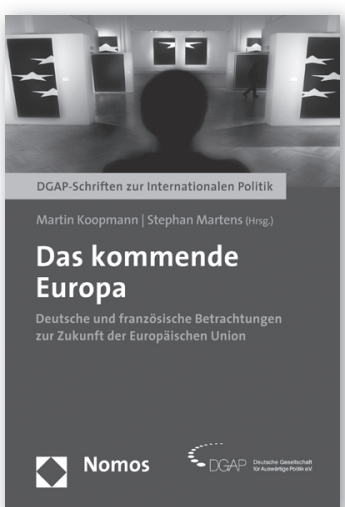

\author{
Das kommende Europa \\ Deutsche und französische Betrachtungen \\ zur Zukunft der Europäischen Union \\ Herausgegeben von Martin Koopmann und \\ Stephan Martens \\ 2008, 388 S., brosch., 49,- €, ISBN 978-3-8329-3265-7 \\ (DGAP-Schriften zur Internationalen Politik)
}

Aus Anlass des 50. Jahrestages der Römischen Verträge verbinden 22 deutsche und französische Autoren eine Bilanz des europäischen Einigungsprozesses mit einer Analyse der künftigen Herausforderungen in Europa. Neben einem Überblick über den Stand der Integration liefert der Band einen Einblick in die deutschen und französischen Wahrnehmungen der Schwerpunkte europäischer Politik.

Bitte bestellen Sie bei Ihrer Buchhandlung oder bei Nomos | Telefon 07221/2104-37 | Fax -43 www.nomos.de | sabine.horn@nomos.de 